\title{
A State-of-health-oriented Power Management Strategy for Multi-source Electric Vehicles Considering Situation-based Optimized Solutions in Real-time
}

\author{
Ahmed M. Ali ${ }^{1}$, Bedatri Moulik ${ }^{2}$, Nejra Beganovic ${ }^{3}$, and Dirk Söffker ${ }^{4}$ \\ ${ }^{1,4}$ Chair of Dynamics and Control, University of Duisburg-Essen, Duisburg, 47057, Germany \\ (1)ahmed.ali@uni-due.de, ${ }^{(4)}$ soeffker@uni-due.de \\ ${ }^{2}$ Department of Electrical and Electronics Engineering, Amity University, 201303 Noida, India \\ bedatri.moulik@amity.edu \\ ${ }^{3}$ Department of Electronics Design, Mid-Sweden University, 85170 Sundsvall, Sweden \\ nejra.beganovic@miun.se
}

\begin{abstract}
This paper presents a novel situation-based power and battery health management strategy for fuel cell vehicles. In such hybrid powertrains, the synergy role of batteries is essential to minimize overall power consumption and maintain higher electric efficiency of the fuel cell. On the other side, lifetime degradation of the battery is associated to the recurrent charging/discharging cycles. The proposed power management strategy addresses the trade-off between these contradictive objectives. Vehicle states in each situation are defined in terms of driver-related characteristic variables (power demand and speed) corporately with a powertrain-related variable (on-board battery's state of charge). Optimal power handling solution for each situation is searched offline considering different optimization criteria: range extension, lifetime maximization, and power consumption minimization. A weighted fusion of these optimized solutions can be implemented online based on desired driving strategy, leading to situation-based optimized solution. This contribution aims to provide flexible power handling options meeting performance requirements (energy efficiency and driveability) without scarifying battery's lifetime. Simulation tests using different driving cycles are conducted for evaluation purpose.
\end{abstract}

\section{INTRODUCTION}

\subsection{Motivation}

Combustion of fossil fuels is one of the major sources of environmental degradation, not only due to related collateral thermal losses but rather due to the harmful exhaust emissions. Besides, the continual decay in global reserves of fossil fuel sets forth the promotion of renewable energy in modern transportation systems as an urgent necessity (Ehsani et al., 2018). Hybrid electric vehicles implement auxiliary power sources to mitigate the dependency on internal combustion engines (ICE). Intelligent hybridization paradigms offer flexible power assist and recuperation among on-board power sources to avoid inefficient operations of the ICE. Such ICE-based hybrids are considered as a short-term solution towards the All-electric vehicle (AEV), achieving zeroemissions. However, preclusion of the ICE is a challenging step, due to the high energy density of gasoline and diesel fuels. Advances in electric storage technologies, i.e. battery and supercapacitor, enabled AEVs to achieve a competitive performance compared to ICE-based vehicles (Onori et al., 2016).

Electric batteries are major components in electrified drivelines. Lithium-Ion (Li-Ion) batteries have higher energy density than capacitors and can take over dynamic power demands better than fuel cells; hence, they are widely implemented in electric drive-lines (Khaligh \& Li, 2010). However, the lifetime of Li-Ion batteries is highly influenced by the intensity of operating conditions, i.a. the power throughput, depth of charge/discharge, and overheating. Nonoptimal scheduling of battery's charging/discharging cycles leads to capacitance fade and decay in the ampere-hour (AH)-throughput. Rapid degradation of the state-of-health (SoH) leads to an earlier replacement of the battery and consequently increases running-costs of the electric vehicle (Kouchachvili et al., 2018).

Power management strategies (PMSs) play an essential role to retain optimal operating conditions in hybrid and electric powertrains. A typical objective of PMSs is to maximize the energy efficiency (mileage per unit energy) of the powertrain by reducing the total power consumption per trip (Silvas et al., 2017). This objective is conflicting with battery's health as it implies the assignment of intense and transient power demands to the battery, which shortens its lifetime and 
leads to an ahead-of-time replacement of such costly component (Moura et al., 2013). Considering the retention of battery's lifetime within the objectives of PMS has a significant potential to achieve the balance between the contradictive goals: instantaneous cost (energy use) and running-cost (battery lifetime) of hybrid powertrains. Moreover, meeting the desired performance requirement (implied by the driver) at various load conditions, without scarifying the targeted objectives, is a challenge in such lifetime-conscious PMSs, that received further attention from researchers in the last few years (Silvas et al., 2017).

\subsection{Previous work}

\subsubsection{Optimal PMS in real-time}

Battery's lifetime-conscious PMSs have been under consistent development to achieve the above-mentioned goals in real-time. Methods of PMSs can be categorized into optimization-based and rule-based ones (Silvas et al., 2017). Optimization-based algorithms are sophisticatedly formulated to define optimal control policies for specific driving cycles. Such algorithms, i.a. dynamic programming or genetic algorithms, are time-intensive and hence are typically used as benchmark solutions to define optimized trajectories for battery's state-of-charge $S o C_{b}$, that achieves minimal health degradation for the considered driving cycle (Ettihir et al., 2016). On the other hand, rule-based algorithms are defined as simple if-else rules and hence are suitable for realtime applications. In such algorithms, experience-based or manufacturer-given knowledge is used to derive the control rules, i.e. charging/discharging boundaries and power assistance/recuperation limits of the battery (Wang et al., 2019).

To enhance the solution optimality of power management decisions in real-time, two main approaches have been presented in literature: first, defining situative rule-based solutions; second, conducting a limited-horizon optimization (Zhang et al., 2015). The first approach implements offline situatively optimized control policies during online application. Vehicle situations, to which optimal solutions are assigned, are defined in terms of multiple characteristic variables, i.a. vehicle speed or $S o C_{b}$. On the other hand, limitedhorizon optimization considers either a simplified powertrain model or an equivalent cost conversion to solve an optimal control problem in real-time. The cost function to be minimized comprises the estimated lifetime degradation and total energy use over the upcoming limited horizon. Kalman filters are widely implemented to estimate future trajectory of $S o C_{b}$ based on approximate battery models, such that estimation errors due to model inaccuracies can be consistently reduced using feedback control (Ali \& Söffker, 2018).

\subsubsection{Modeling of Battery degradation}

Battery degradation models in literature have been presented in two ways: first, analytical models of the physical-chemical reactions within battery cells have been developed to acquire more insight into capacity fade mechanisms and the sources of battery aging (Lipu et al., 2018). From an electrochemical perspective, emulating the change in solid electrolyte interface (SEI) film thickness is a typical example of such models (Moura et al., 2013). Full knowledge of modeling parameters is essential for such models, which is either intricate to yield or time-consuming to estimate (Bashash et al., 2010). Second, empirical and semi-empirical aging models are used based on generic simplified relations to be fitted to related experimental results (Yue et al., 2019). These are simple, yet accurate models and hence widely implemented in lifetimeconscious PMSs. Such models are based on emulating three main physical phenomena: 1) capacity fade, 2) increase of internal resistance, and 3) decay in remaining lifetime of the battery.

Fade of capacity refers to the battery's inability to hold the same amount charge after repeating cycles or usage, as usually batteries are replaces when no longer capable of holding $20-30 \%$ of their nominal capacitance (Yue et al., 2019). Furthermore, a merge between ohmic equivalent circuits and empirical relations can be used to express the change in battery's internal resistance (Remmlinger et al., 2011). Factors believed to influence the degradation process of batteries can be summarized as: operating temperature, allowable boundaries for $S o C_{b}$, depth-of-discharge (DOD), high current throughput, recharging time(s), and storage conditions (humidity, duration, etc.) (Yue et al., 2019). Due to the lack of crisp analytical interrelations between such factors, empirical modeling of battery's degradation became indispensable to an accurately assess between these factors. Therefore, empirical battery degradation models has been increasingly considered in lifetime-conscious PMSs due to the ease of implementation and tuning requirements of such models (Hoke et al., 2011; Fotouhi et al., 2018).

\subsection{Problem statement and contribution}

Although the balance between battery degradation and energy efficiency is considered as an optimal solution to lifetimeconscious PMSs, inconsideration of driver's demand may degrade the applicability of such solutions (Yue et al., 2019). Providing optimized power management solutions that meet variable driver requirements, i.e. extended mileage or persistent accelerations, with minimal mitigation of battery's lifetime is less introduced in literature (Lipu et al., 2018). Such situation-based PMSs have a significant potential to accommodate unscheduled driving loads without scarifying the desired optimality.

This contribution proposes a situation-based PMSs that de- 
fines optimal power handling decisions for different driving situations. The battery degradation is represented to the cost function as an empirical residual lifetime model. Five cases of cost function minimization are solved, considering gradual change of the weighting factors between the objectives: energy efficiency and lifetime degradation. The optimized solutions for these cases are used to generate a Pareto front for the optimization problem. For real-time application, the optimized solutions can be merged to flexibly fulfill different driver's demand and retain the Pareto optimality.

This contribution is organized as follows: in chapter 2, the powertrain configuration and parameters modeling are presented. The lifetime-oriented PMS is explained in chapter 3. Simulation results, analysis, and discussion are given in chapter 4 , followed by the conclusion and future work in chapter 5 .

\section{Powertrain Configuration}

The implemented powertrain in this study is an all-electric hybrid one, that comprises a fuel cell (FC), battery (B), and supercapacitor (SC) as shown in Fig. 1. The power sources are set up in a series-parallel topology, whereby the fuel cell is the primary power source and both the battery and supercapacitor are auxiliary ones. Each power source is coupled with an inline DC/DC converter to retain a unified voltage $u_{\text {bus }}$ at the load side. This drive-line topology is investigated to achieve optimal performance for fuel cell hybrid powertrains (Özbek et al., 2013).

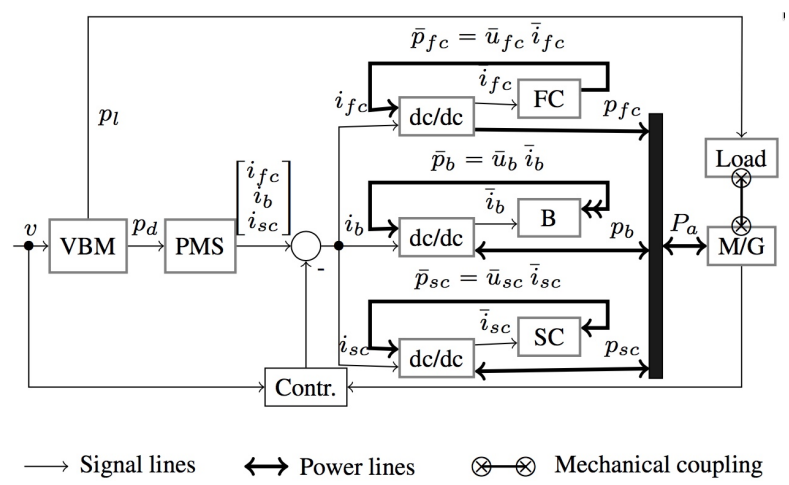

Figure 1. Driveline topology and operating principle of the fuel cell hybrid electric powertrain.

Modeling of powertrain operation is based on calculating traction power demand $p_{d}$ within the vehicle backward model (VBM) given the desired speed (from driving cycle) as

$$
\begin{aligned}
p_{d} & =f_{x} v \\
& =(m a+\underbrace{\frac{A \rho C_{d}}{2}\left(v-v_{d}\right)^{2}}_{\text {Air drag }}+\underbrace{m g \sin \theta}_{\text {Grade res. }}+\underbrace{\mu m g \cos \theta}_{\text {Rolling res. }}) v,
\end{aligned}
$$

where $f_{x}$ denotes traction force, $m g$ vehicle curb weight, $a$ longitudinal acceleration, $\rho$ air density, $A$ vehicle frontal area, $C_{d}$ air drag coefficient, $v_{d}$ wind speed, $\mu$ rolling resistance coefficient, and $\theta$ is the road grade. Online power handling strategies are decided by the situation-based PMS. Besides, a supervisory control module is implicitly defined in the PMS to ensure the operation within safe limits of individual powertrain components.

The supervisory control is programmed in state machine language as shown in Fig. 2. The states are grouped based on three main modes of operation: power idle, demand, and recuperation. The inputs $p_{d}, S o C_{b}$, and the supercapacitor state-of-charge $S o C_{s c}$ are used to define the state transitions within each mode. The operating boundaries to be retained are $S o C_{b}^{\min }, S o C_{b}^{\max }, S o C_{s c}^{\min }, S o C_{s c}^{\max }$, and $p_{f c}^{\max }$. Optimized power handling decisions not conforming to these boundaries are overridden by the supervisory control and excluded during offline optimization process. The demanded power from each source $p_{f c}, p_{b}$, and $p_{s c}$ are defined as

$$
\begin{aligned}
p_{f c} & =u_{f c} i_{f c}, \\
p_{b} & =u_{b} i_{b}, \text { and } \\
p_{s c} & =u_{s c} i_{s c},
\end{aligned}
$$

such that

$$
u_{f c} \approx u_{b} \approx u_{s c} \approx u_{b u s}
$$

which implies that desired output current from each source $\left[\begin{array}{lll}i_{f c} & i_{b} & i s c\end{array}\right]^{T}$ represent the control signal to respective DC/DC converter. Operation of the DC/DC converter can be simplified as

$$
i_{\text {in }}=i_{\text {out }} \frac{c}{u_{\text {in }}} \frac{1}{\mu_{\text {conv }}},
$$

where the suffices ${ }_{i n}$ and out denote input and output current and voltage, $c$ is a constant representing desired bus voltage, and $\mu_{c o n v}$ is the tabulated conversion efficiency based on experimental validation of the simplified model (Özbek et al., 2013). The output $i_{\text {out }}$ in each DC/DC converter becomes $\bar{i}_{f c}, \bar{i}_{b}$, and $\bar{i}_{s c}$ for the fuel cell, battery, and supercapacitor accordingly. Fuel cell voltage can be calculated considering activation-, ohmic-, and concentration-voltage losses, $u_{\text {act }}$, $u_{\text {ohm }}$, and, $u_{\text {conc }}$, as

$$
\begin{aligned}
u_{f c}=n_{c}\left(u_{f c}^{o}\right. & -\underbrace{\left(c_{1}+c_{2}\left(1-e^{-i_{f c} c_{3}}\right)\right)}_{u_{a c t}} \cdots \\
& -\underbrace{i_{f c} R_{f c} \cdots}_{u_{\text {ohm }}} \\
& -\underbrace{\left.i_{f c}\left(\frac{c_{4} i_{f c}}{i_{\text {max }}}\right)^{c_{5}}\right)}_{u_{\text {conc }}},
\end{aligned}
$$



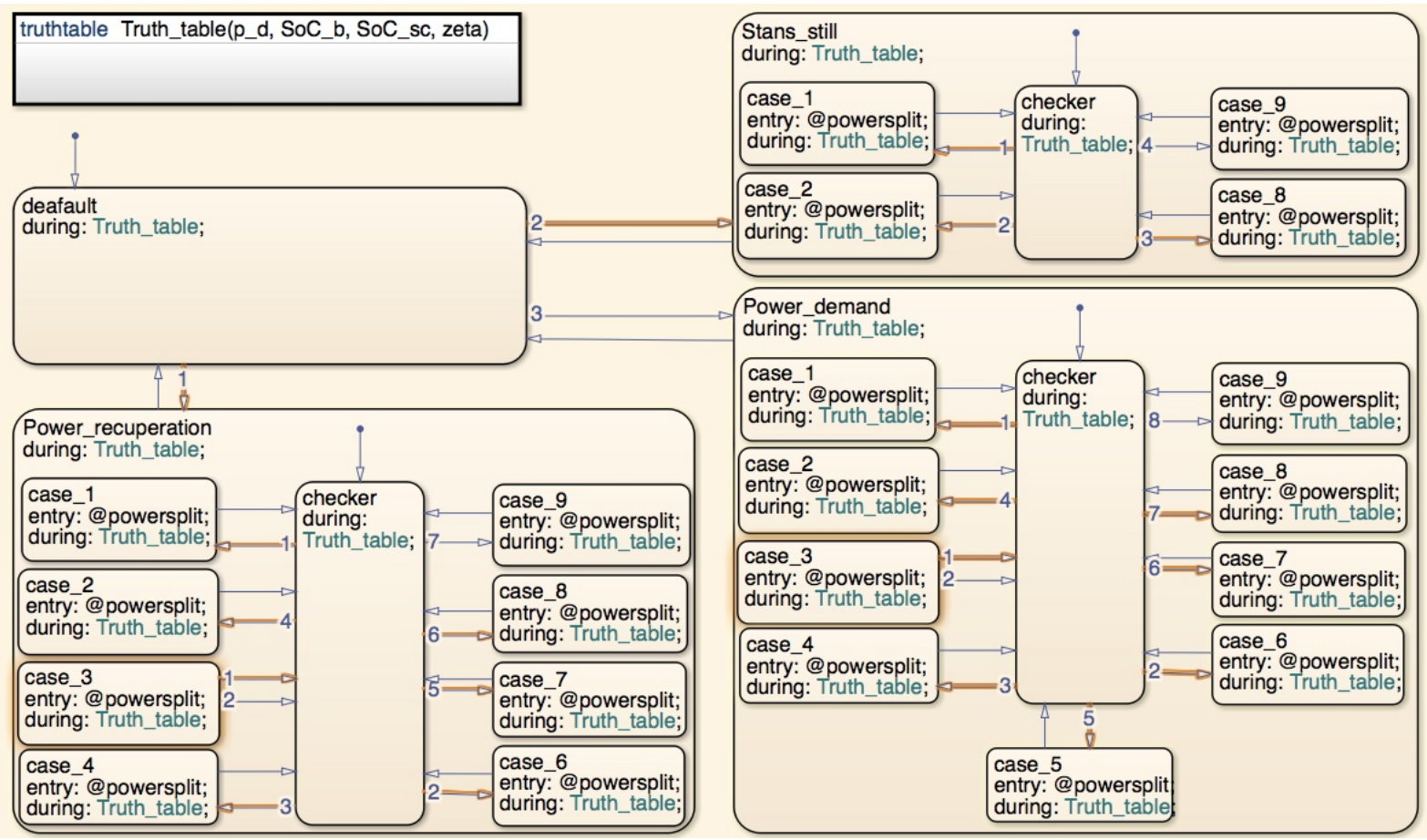

Figure 2. Supervisory control as a state machine program

where $n_{c}$ denoted the number of cells in the stack, $u_{f c}^{o}$ the open circuit voltage, $c_{1}, \ldots, c_{5}$ constants based on experimental validation, $R_{f c}$ the internal resistance of the fuel cell, and $i_{\max }$ is the maximum delivery current (Özbek et al., 2013).The supercapacitor is modeled as

$$
u_{s c}=u_{s c}^{o}-R_{s c} i_{s c}-\frac{1}{c_{s c}} \int_{t_{i}}^{t_{f}} i_{s c} d t,
$$

where $u_{s c}^{o}$ is the open circuit voltage, $R_{s c}$ and $C_{s c}$ are equivalent capacitance and internal resistance of the supercapacitor accordingly, and $t_{i}$ and $t_{f}$ are initial and final time of an infinitesimal simulation step. Both $C_{b}$ and $C_{s c}$ are statically modeled neglecting the capacitance loss due to components' aging and lifetime degradation. The state of charge of $(\mathrm{SoC})$ is calculated as

$$
S o C=S o C_{i}-\frac{1}{Q_{n o m}} \int_{t_{i}}^{t_{f}} I d t,
$$

where $S o C_{i}$ is the initial state of charge, $Q_{n o m}$ the rated capacity, and $I$ is either $i_{b}$ or $i_{s c}$. The actual power delivery $p_{a}$ at motor terminals should conform to calculated power demand $p_{d}$ considering conversion losses as

$$
\begin{aligned}
p_{a} & =p_{f c}+p_{b}+p_{s c} \\
& =\bar{p}_{f c}+\bar{p}_{b}+\bar{p}_{s c}+\text { losses },
\end{aligned}
$$

so that $p_{f c}, p_{b}$, and $p_{s c}$ are determined based on the split ratio

$$
\begin{aligned}
& \psi=\left[\begin{array}{ll}
\beta & \gamma
\end{array}\right] \text { as } \\
& \quad\left[\begin{array}{lll}
p_{f c} & p_{b} & p_{s c}
\end{array}\right]^{T}=\left[\begin{array}{lll}
\beta & \gamma & 1-(\beta+\gamma)
\end{array}\right]^{T} p_{a},
\end{aligned}
$$

subjects to the constraint

$$
\beta+\gamma \leqslant 1, \quad \forall\{\beta, \gamma\} \in[0,1] .
$$

The motor/generator $(\mathrm{M} / \mathrm{G})$ is mechanically coupled to a programmable load-motor emulating the load $p_{l}$ in terms of the rolling resistance and air drag according to the speed input $v$. Modeling of the Li-ion battery is based on the equivalent circuit as

$$
u_{b}=u_{b}^{o}-R_{b} i_{b}-\frac{1}{C_{b}} \int_{t_{i}}^{t_{f}} i_{b} d t
$$

where $u_{b}^{o}$ is the open circuit voltage and $R_{b}$ and $C_{b}$ are battery's capacitance and internal resistance accordingly. Capacitance loss of the battery $Q_{\text {loss }}$ is simply considered proportional to the number of charging/discharging cycles $n_{\text {cycles }}$ as

$$
Q_{\text {loss }}=c_{6} n_{\text {cycles }},
$$

where, the change in $u_{b}^{o}$ and $C_{b}$ due to $Q_{\text {loss }}$ is statically defined within look-up tables (MATLAB, 2019). Residual lifetime of the battery $L_{r e s}$ is estimated empirically considering the operating temperature $T_{b}$, the depth-of-discharge $D o D$, and the power throughput $p_{b}$ according the work presented 
in (Hoke et al., 2011) as

$$
L_{r e s}=f\left(Q_{\text {loss }}, T_{b},\left(1-S o C_{b}\right), p_{b}, c_{7} \ldots c_{10}\right),
$$

where the constants $c_{6}-c_{10}$ are tuned such that $L_{r e s}$ matches a nominal lifetime $L_{n o m}$ at $10^{4}$ cycles before reaching to $Q_{\text {loss }}=80 \%$. The considered battery degradation model has the following limitation: First, $n_{\text {cycles }}$ is calculated considering only complete cycles from maximum to minimum $S o C_{b}$; second, the effect of ambient temperature on $T_{b}$ is neglected; third, the uncertainty of linearly anticipated $L_{r e s}$ is omitted. However, such this model has been plausibly implemented in many lifetime-conscious PMSs, where the main objective is to define optimal control strategy considering the trade-off between energy efficiency and battery degradation (Fotouhi et al., 2018).

\section{BATTERY'S LIFETIME-ORIENTED PMS}

\subsection{Situation-based PMS}

Situation-based PMSs are based on defining specific vehicle states, to which the control parameters can be optimized (Gong et al., 2008). Vehicle states can be defined heuristically or based on multiple characteristic variables from driving data history. For online application, vehicle states are recognized in real-time and the optimized solutions can be assigned accordingly. Situation-based PMSs are proved to have low computational requirements and being capable of yielding near-optimal solutions for the power management problem. An illustration of the working principle of situation-based PMS is given in Fig. 3. In this contribution,

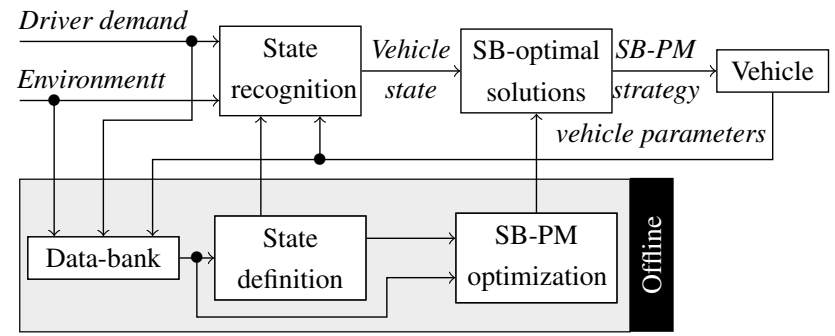

Figure 3. Operating principle of situation-based power management system.

a multi-dimensional space (grid-space) is defined for linear mapping of vehicle states. The axes of grid-space are the selected characteristic variables, to which specific discretization levels are assigned. An exemplification of alternative structures of grid-space is shown in Fig. 6. In a previous work of the authors (Ali et al., 2019), the impact of different characteristic variables on total cost minimization is statistically analyzed, putting forth that certain constellations (set of axis at certain discretization levels) outperforms all other constellations at various driving cycles. This contributions proceeds with a constellation comprising the variables: vehicle speed on $\mathrm{V}$-axis, power demand on P-axis, and $S_{o} C_{b}$ on B-axis.
Numerical values for discrete levels of grid-space axes are

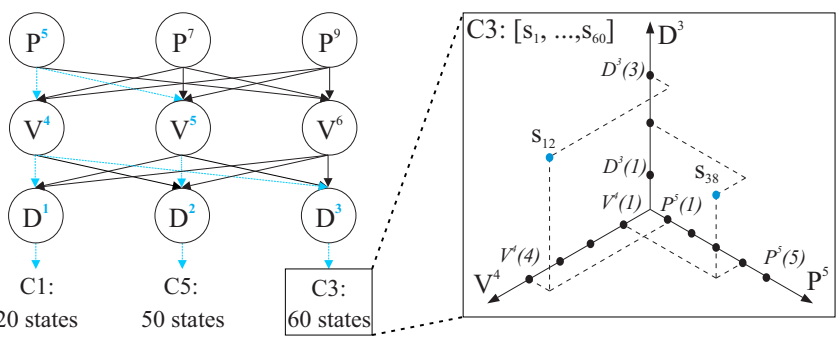

Figure 4. Implementation of characteristic variables in grid-space structure with focus on state definition in an exemplified constellations C3.

subject to an optimization process that aims to improve the representation of all vehicle states in grid-space. This can be explained, for instance, for the $\mathrm{V}$-axis as

$$
V^{n}=\left[V_{1}, V_{2}, \ldots, V_{n}\right],
$$

is the vector comprising $n$ discrete levels for vehicle speed. The optimization task is defined as

$$
\begin{aligned}
& \underset{\text { s.t. }}{\operatorname{minimize}} \quad \mathbf{J}_{1}=f\left(\mathrm{~N}_{v}, V^{n}\right), \\
& \qquad \mathbf{V}^{n}(i) \in\left[\begin{array}{ll}
v_{\min } & v_{\max }
\end{array}\right],
\end{aligned}
$$

where $N_{v}$ denotes points' count of $v$ in the data-bank, $f$ a function defining the difference in points' count between the intervals $\left[V_{i}^{n}: V_{i+1}^{n}\right]$, where $i=1,2, \ldots, n$ as shown in Fig. 5. This optimization process is conducted simultaneously for the other P- and B-axes. The acquired achievement by solving Eqn. (17a) is shown in Fig. 6, where more homogeneous representation and less number of missed stated could be achieved in the optimally discretized grid-space.

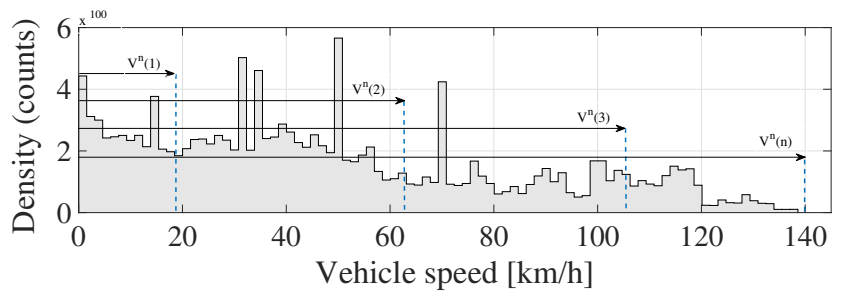

Figure 5. Projection of discrete values for $\mathrm{V}^{n}$ on the histogramic plot of vehicle speed in the data-base.

Optimal power split ratio for each vehicle state is defined as

$$
\Psi=\left[\begin{array}{llll}
\psi_{1} & \psi_{2} & \ldots & \psi_{m}
\end{array}\right]
$$

where $\psi_{i}$ denotes the split ratio associated to respective vehicle state $s_{i} \forall i \in\{1,2, \ldots, m\}$, where $m$ is the total number of vehicle states in grid-space. The optimization task is defined as

$$
\min \quad \mathbf{J}=\min \left[\begin{array}{ll}
\alpha_{1} & \alpha_{2}
\end{array}\right]\left[\begin{array}{l}
o b j_{1}(\Psi, x, t) \\
o b j_{2}(\Psi, x, t)
\end{array}\right],
$$




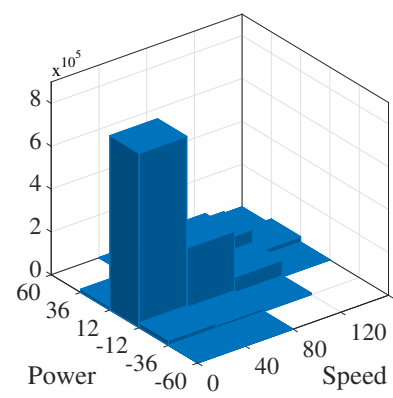

(a)

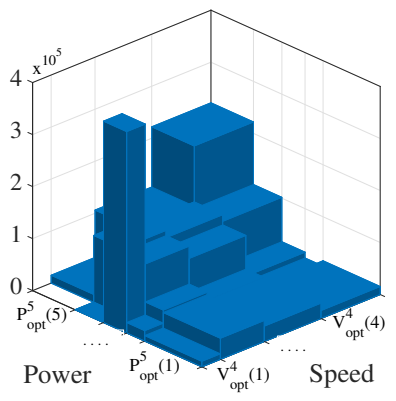

(b)
Figure 6. Improving vehicle states representation in grid-space using optimally-spaced axes in (6b) compared to equally-spaced ones in (6a).

for

$$
\begin{aligned}
& \operatorname{obj}_{1}(\Psi, x, t)=\int_{t_{0}}^{t_{f}} p_{f c} d t \quad \text { and } \\
& \operatorname{obj}_{2}(\Psi, x, t)=\int_{t_{0}}^{t_{f}} L_{\text {loss }} d t
\end{aligned}
$$

s.t.

$$
\begin{aligned}
S o C_{b}^{\text {min }} & <S o C_{b}<S o C_{b}^{\max }, \\
S o C_{b}^{\text {min }} & <S o C_{b}<S o C_{b}^{\max }, \\
i_{f c}^{\text {min }} & <i_{f c}<i_{f c}^{\max }, \\
i_{b}^{\min } & <i_{b}<i_{b}^{\max }, \\
i_{s c}^{\text {min }} & <i_{s c}<i_{s c}^{\max }, \text { and } \\
\beta+\gamma & \leqslant 1, \quad \forall\{\beta, \gamma\} \in[0,1],
\end{aligned}
$$

where $t_{0}$ and $t_{f}$ denote initial and final simulation time, $\alpha_{1}$ and $\alpha_{2}$ weighting factors, $L_{\text {loss }}=$ the lifetime degradation of the battery $\left(L_{n o m}-L_{r e s}\right)$, and $x$ the state vector defined as

$$
x=\left[\begin{array}{lllllll}
i_{f c} & i_{b} & i_{s c} & Q_{l} \text { oss } & T & S o C_{b} & S o C_{s c}
\end{array}\right] .
$$

The formulation of objectives $o b j_{1}$ and $o b j_{2}$ sets forward the conflict between minimizing the $p_{f c}$ and $L_{r e s}$ simultaneously, whereby the conventional lifetime-oriented strategy by minimizing $i_{b}$, is not sufficient to achieve the balance between both objectives. The optimization problem is a typical multi-objective one, solved as a weighted-sum minimization to introduce interactive effect through different values for the weighting factors $\alpha_{1}$ and $\alpha_{2}$.

\section{RESULTS ANALYSIS AND DISCUSSION}

\subsection{Offline optimization results}

Five different cases are assigned to solve Eqn. (19), based on the values of $\alpha_{1}$ and $\alpha_{2}$ shown in Table 1. The optimization cases vary from highest priority for battery's health in case1 up to highest priority for minimizing fuel cell energy in case-5. The cost minimization problem in each case is solved using non-dominant sorting genetic algorithms (NSGA-II), based on the work presented in (Deb et al., 2002; Lin, 2011). This contribution considers 100 generations, each of 50 populations to acquire the optimal solution using NSGA-II. The mutation and crossover rates are adaptively set based on the size of explored space. The driving cycle used for offline optimization is based on standard regulations for abuse testing of Li-ion batteries as shown in Fig. 7 (Ruiz et al., 2018).

Table 1. Values of the weighting factors for each optimization case.

\begin{tabular}{|c|c|c|c|c|c|}
\hline & Case-1 & Case-2 & Case-3 & Case-4 & Case-5 \\
\hline$\alpha_{1}$ & 1 & 3 & 5 & 7 & 9 \\
\hline$\alpha_{2}$ & 9 & 7 & 5 & 3 & 1 \\
\hline
\end{tabular}

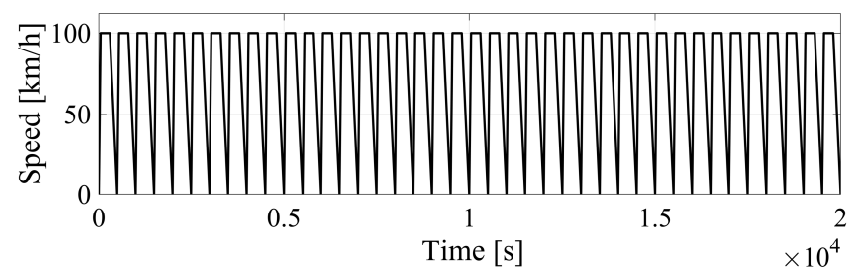

Figure 7. Speed profile during offline optimization showing repeating acceleration, coasting, and deceleration rounds for 5.56 hours.

Optimization results for the cost function, including all individual solutions from the first generation and the evolved best solution from each generation up to the end are shown in Fig. 8. The significance of random exploration of the solution space can be perceived from the figure, leading to rapid converge to the global. Moreover, given a normalized measure for both objectives during the optimization, it can be noticed that penalizing inefficient usage of the battery leads to better values of total cost in cases 1-3. Contrarily, reducing these penalties in cases 4 and 5 results in relative increase of the total cost. This point is discussed quantitatively in the sequel. Objectives-wise, the direction of solution's evolution towards optimality can be contextually explained, given the weighting factors for each case (see Table 1). In case-1, a vertical evolution can be notices (minimizing $o b j_{2}$ ), towards a gradually horizontal advance in case-5 (minimizing $o b j_{1}$ ), meeting the optimality criterion in each case. The best ten solutions in each case are shown in Fig. 9. While the ordinate of all solutions almost cover the whole span of $o b j_{2}$, their abscissa falls $\approx 50 \%$ of the achievable span for $o b j_{1}$, which points out the minimal requirements on the fuel cell as a primary power source. Besides, a patent vicinity can be identified between the solutions in cases 1 and 2, where $\alpha_{2}$ still outweighs $\alpha_{1}$, which promotes battery health concerns in $o b j_{2}$. A pseudo Pareto front is established for this control problem using curve fitting tools based on available results.

\subsection{Online application and comparative evaluation}

For the online testing and evaluation of developed situationbased health-conscious power management system (SB-HC), 

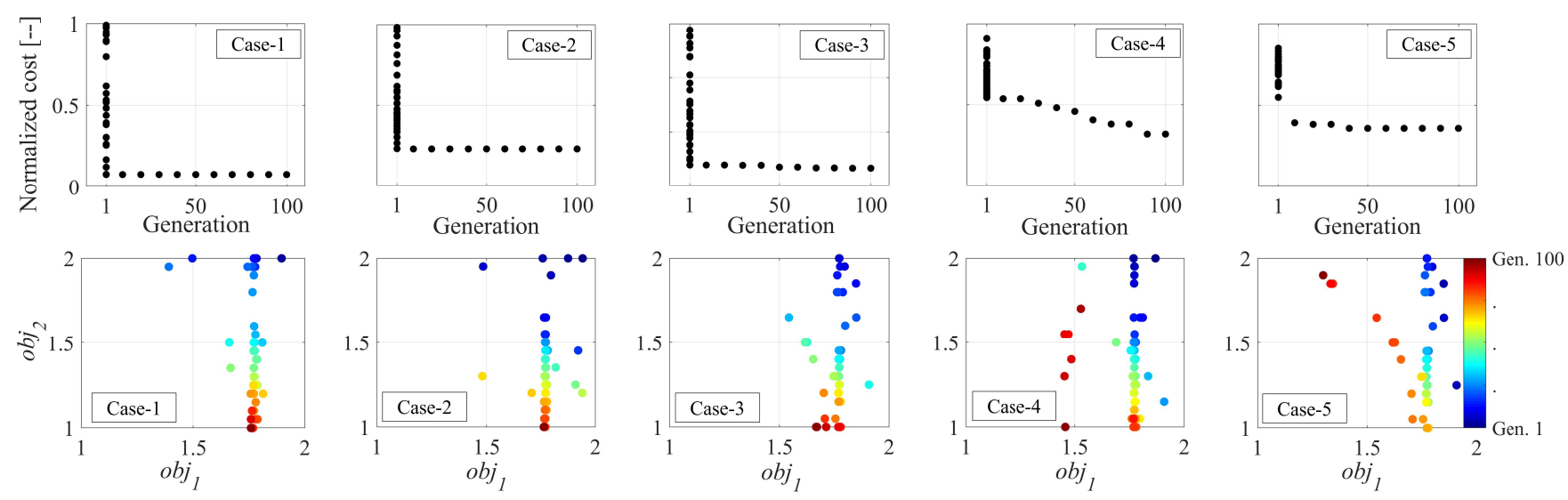

Figure 8. Evolution of the cost function minimization through generations and iterations using NSGA-II.

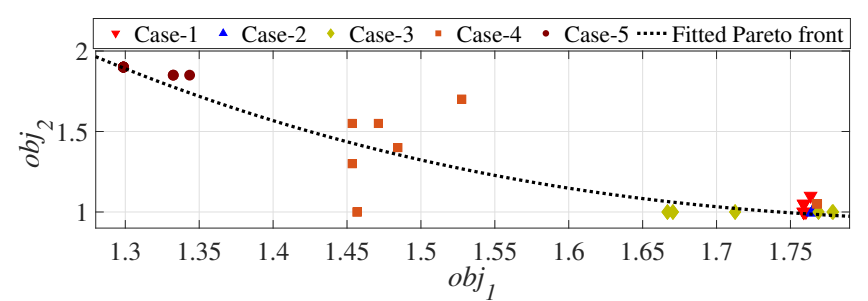

Figure 9. Fitted curve for the optimality Pareto front of the cost minimization problem based on best results in each case.

nine repeated US06 driving cycles have been considered. For each of these driving cycles, one of the optimized solutions or a merge between two solutions have been applied as shown in Fig. 10. This scheme aims to emulate different driver's attitudes, to which optimized solutions can be flexibly assigned to retain battery's lifetime without scarifying the driveability. The developed method is comparatively evaluated against a single-case optimized rule-based one, which implements no weighting factors into the rules optimization. The rec-

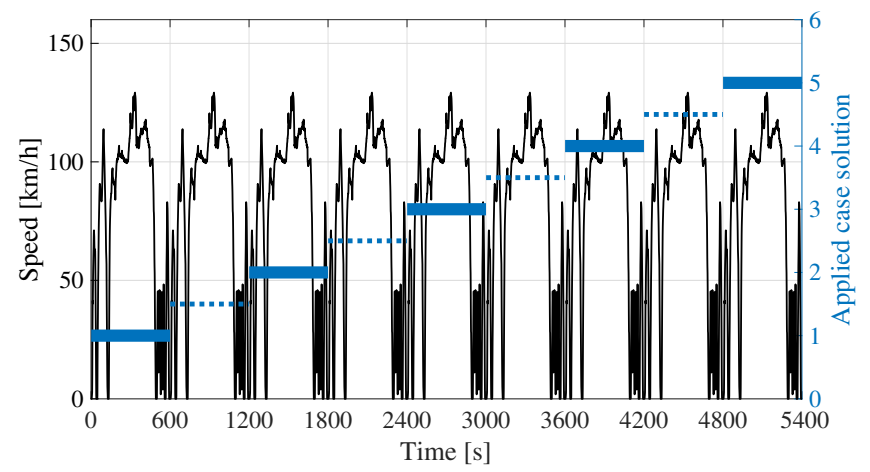

Figure 10. Applying different control strategies over nine repeated us06 driving cycles; dashed lines for merged solutions between two consequent cases.

ognized vehicle states during online application are shown in Fig. 11. The implemented structure of grid-space offers 324 discrete states based on the characteristic variables: ve- hicle speed, power demand, and $S o C_{b}$. As the former two variables are not control-dependent, i.e. are identical in both control methods, the difference in recognized states should be only a function of $S o C_{b}$. Having the same initial conditions, both methods achieved the same states almost up to the end of first cycle (second 500). Afterwards, different states are achieved for each method, to which totally different solutions have been optimized offline. Approaching the eighth cycle (second 4000), vehicle states started to match again. Applied control cases in the last two cycles are less protective regarding battery's lifetime aspects, which retains $S o C_{b}$ into similar working regions as per the RB method. The tran-

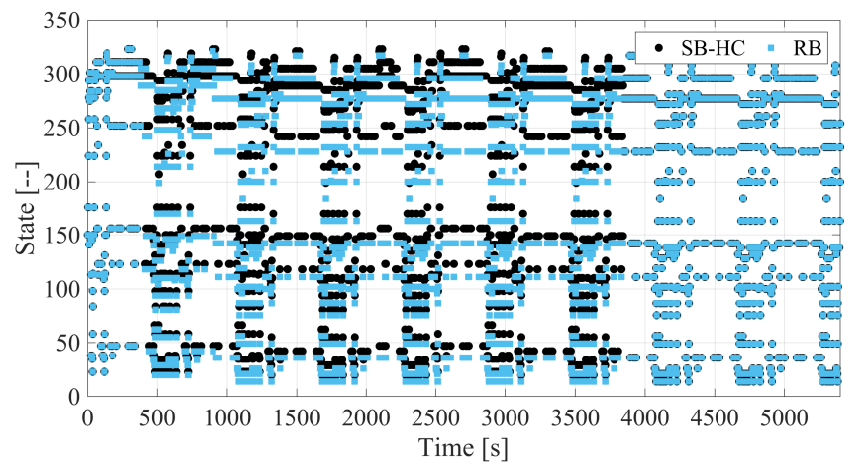

Figure 11. Vehicle states, from grid-space, for both control methods: SB-HC and RB.

sitions of vehicle states are explained in detail showing the $S_{o} C_{b}$ and $S o C s c$ profiles for both control methods in Fig. 12 . It can be seen in Fig. 12 that RB method performs a moderate charge-sustaining strategy in almost three consequent us06 cycles before reaching the lower limit for $S o C_{b}$. On the other side, the SB-HC algorithm conducts a conservative strategy at the beginning, preserving the on-board charge longer and avoiding higher DoD values. The impact of each control strategy on the synergy role of the supercapacitor can be explained from the results shown in Fig. 12. The rapid depletion of $S o C_{b}$ using $\mathrm{Rb}$ method put more synergy tasks on the supercap., i.e. several cycles and accordingly unsched- 
uled loading of the fuel cell. The power output profile of the

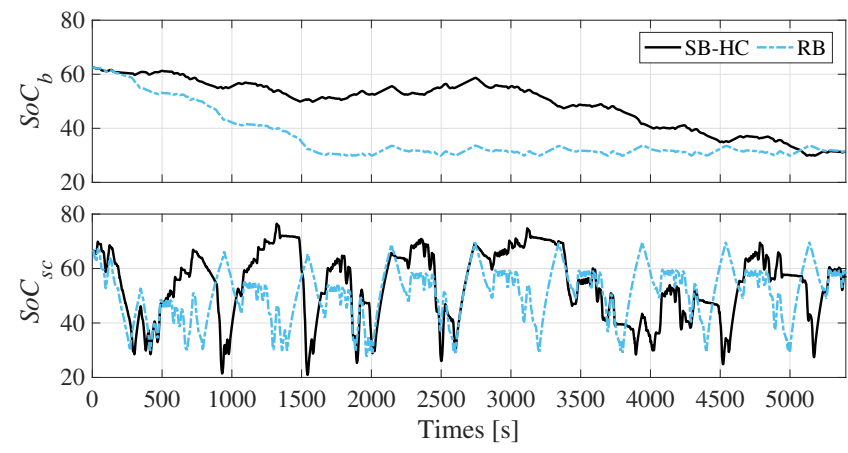

Figure 12. State of charge profiles for the battery and supercapacitor.

battery as shown in Fig. 13 reveals more information about each control strategy, where the faster charge depletion using RB method urged repeating recharging phases to retain $S o C_{b}$ above the lower boundaries. It can be seen that relatively more recharging power has been assigned to the battery using RB method more than SB-HC. This implicitly requires unscheduled power from the fuel cell and urges the supervisory control to override the offline optimized solution, i.e. temporarily violate the optimal trajectory.

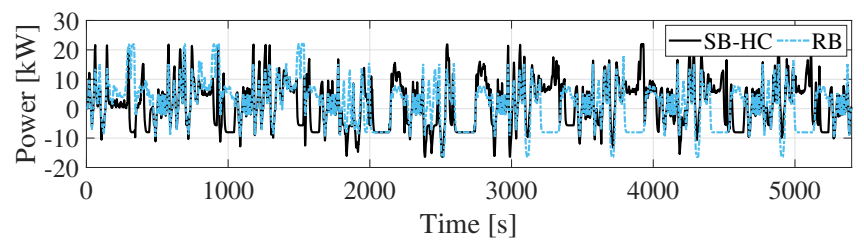

Figure 13. Power throughput of the battery using both PMSs.

To get more insight into the operation of the fuel cell and the estimated battery degradation, accumulated $p_{f c}$ and $\Delta L_{b}$ are shown in Fig. 14. The accumulated $\Delta L_{b}$ in the figures is proportionally related to the above shown results for $T . p_{b}$, and $S o C_{b}$. It is presumable, that prioritizing battery's health will induce an increase in fuel cell energy consumption, which reached for the overall test to $11.15 \%$. However, the reduction in lifetime degradation is substantial $\approx 64.63 \%$. Due

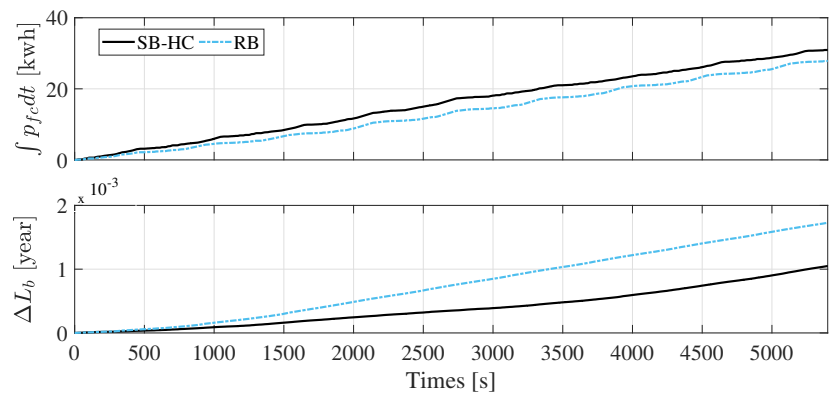

Figure 14. Total consumed energy and accumulated drop in residual lifetime using SB-HC and RB methods.

to the fact that multiple cases within the developed SB-HC method are applied during the same test, it is more relevant to analyze the previous energy and battery degradation results for cycle-based segments as shown in Fig. 15. From the results, it can be concluded that using SB-HC method led to an increasing consumption of fuel cell energy per cycles in the first five cycles, i.e. where the battery's lifetime has been prioritized. However, in three out of the last four cycles, SB-HC method assigned less synergy role to the fuel cell. Regarding the battery's lifetime degradation per cycle, the SB-HC method achieved steadily lower $\Delta L_{b}$ per cycle for the whole test procedures. The last four cycles show that SB-HC method can achieve total energy saving and yet preserve battery's lifetime. This puts forth the impact of retaining battery's health and flexibly responding to variable driver's demands, which is the core of this contribution. In
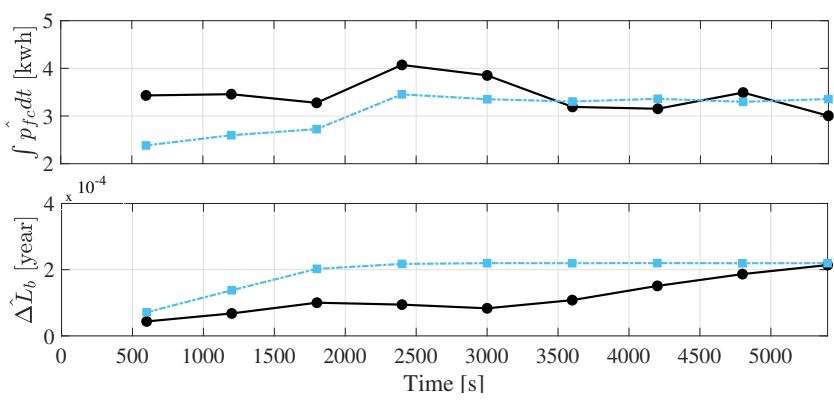

Figure 15. Change per one driving cycle in total consumed energy and accumulated drop in residual lifetime for both control methods.

Table 2, a descriptive summary of the energy efficiency and battery's lifetime results is presented. Energy efficiency $\left(\eta_{e n}\right)$ is a well-known measure for evaluating vehicular propulsion efficiency, based on consumed energy per $100 \mathrm{~km}$. In the table, $\eta_{e n}$ is calculated per cycle to evaluate the energy consumption and the total value as well. Summarized results of battery's lifetime $\Delta L_{b}$ reveal the significant reduction in battery degradation over individual cycles, i.e. various driver's behavior for the whole trip.

\section{CONCLUSION AND FUTURE STEPS}

In this contribution, a novel method addressing battery degradation issues in power management of hybrid electric vehicles is presented. The method implements a situation-based principle to assign optimized power handling solutions in different vehicle states in real-time. Vehicle states are defined in terms of multiple characteristic variables, i.e. vehicle speed, power demand, and $S o C_{b}$. The power management problem for the fuel cell hybrid vehicle is defined in terms of minimizing two objectives: consumed energy from the fuel cell, and battery's degradation. The optimization problem is solved offline as a weighted-sum using NSGA-II.

For the offline optimization, five cases are assigned to the weighting factors, to define the optimized solutions at dif- 
Table 2. Summary of online test results for energy efficiency and battery's lifetime.

\begin{tabular}{|c|c|c|c|c|c|c|c|c|c|c|c|}
\hline & \multicolumn{9}{|c|}{ Per cycle nr. } & \multirow{2}{*}{$\begin{array}{c}\text { Total } \\
-\end{array}$} \\
\hline & & 1 & 2 & 3 & 4 & 5 & 6 & 7 & 8 & 9 & \\
\hline \multirow{2}{*}{$\eta_{e n}[\mathrm{kwh} / 100 \mathrm{~km}]$} & SB-HC & 26.8 & 27.0 & 25.6 & 31.8 & 30.1 & 24.9 & 24.6 & 27.3 & 23.5 & 29.7 \\
\hline & $\mathrm{RB}$ & 18.6 & 20.3 & 21.3 & 27.0 & 26.2 & 25.8 & 26.3 & 25.8 & 26.2 & 26.7 \\
\hline \multirow{2}{*}{$\Delta L_{b}\left[\mathrm{x} 10^{-5}\right.$ year $]$} & SB-HC & 4.36 & 6.78 & 10.0 & 9.44 & 8.32 & 10.8 & 15.1 & 18.7 & 21.4 & 104.9 \\
\hline & $\mathrm{RB}$ & 7.14 & 13.8 & 20.2 & 21.7 & 22.0 & 22.0 & 22.0 & 21.9 & 22.0 & 172.1 \\
\hline
\end{tabular}

ferent priority levels of each objective. These solutions can be decisively selected by the driver or adaptively set according to the battery's status. Results from the online application reveals the ability of presented method to accommodate different driver's priorities during the whole trip, and yet achieve a significant improvement in preserving battery lifetime. An increase of $11 \%$ in the fuel cell's energy is yielded using SB-HC; however, an improvement up to $60 \%$ in battery's residual lifetime could be achieved.

\section{REFERENCES}

Ali, A. M., Shivapurkar, R., \& Söffker, D. (2019). Optimal situation-based power management and application to state predictive models for multi-source electric vehicles. IEEE Transactions on Vehicular Technology, submitted.

Ali, A. M., \& Söffker, D. (2018). Towards optimal power management of hybrid electric vehicles in real-time: A review on methods, challenges, and state-of-the-art solutions. Energies, 11(3). doi: 10.3390/en11030476

Bashash, S., Moura, S. J., \& Fathy, H. K. (2010). Charge trajectory optimization of plug-in hybrid electric vehicles for energy cost reduction and battery health enhancement. In Proceedings of the 2010 American Control Conference. doi: 10.1109/acc.2010.5530497

Deb, K., Pratap, A., Agarwal, S., \& Meyarivan, T. (2002). A fast and elitist multiobjective genetic algorithm: NSGA-II. IEEE Transactions on Evolutionary Computation, 6(2), 182-197. doi: 10.1109/4235.996017

Ehsani, M., Gao, Y., Longo, S., \& Ebrahimi, K. (2018). Modern electric, hybrid electric, and fuel cell vehicles, third edition. Boca Raton: CRC Press. doi: 10.1201/9780429504884

Ettihir, K., Boulon, L., \& Agbossou, K. (2016). Optimization-based energy management strategy for a fuel cell/battery hybrid power system. Applied Energy, 163, 142-153. doi: 10.1016/j.apenergy.2015.10.176

Fotouhi, A., Propp, K., Auger, D. J., \& Longo, S. (2018). State of charge and state of health estimation over the battery lifespan. In Behaviour of lithium-ion batteries in electric vehicles (pp. 267-288). Springer International Publishing. doi: 10.1007/978-3-319-69950-9-
11

Gong, Q., Li, Y., \& Peng, Z.-R. (2008). Trip based optimal power management of plug-in hybrid electric vehicle with advanced traffic modeling. SAE International Journal of Engines, 1(1), 861-872. doi: 10.4271/200801-1316

Hoke, A., Brissette, A., Maksimovi, D., Pratt, A., \& Smith, K. (2011). Electric vehicle charge optimization including effects of lithium-ion battery degradation. In 2011 IEEE Vehicle Power and Propulsion Conference (p. 18). doi: 10.1109/VPPC.2011.6043046

Khaligh, A., \& Li, Z. (2010). Battery, ultracapacitor, fuel cell, and hybrid energy storage systems for electric, hybrid electric, fuel cell, and plug-in hybrid electric vehicles: State of the art. IEEE Transactions on Vehicular Technology, 59(6), 2806-2814. doi: 10.1109/TVT.2010.2047877

Kouchachvili, L., Yaci, W., \& Entchev, E. (2018). Hybrid battery/supercapacitor energy storage system for the electric vehicles. Journal of Power Sources, 374, 237-248. doi: 10.1016/j.jpowsour.2017.11.040

Lin, S. (2011). NGPM - A NSGA-II Program in Matlab v1.4. Retrieved from de.mathworks.com/ matlabcentral /fileexchange

Lipu, M. H., Hannan, M., Hussain, A., Hoque, M., Ker, P. J., Saad, M., \& Ayob, A. (2018). A review of state of health and remaining useful life estimation methods for lithium-ion battery in electric vehicles: Challenges and recommendations. Journal of Cleaner Production, 205, 115-133. doi: 10.1016/j.jclepro.2018.09.065

MATLAB. (2019). Nonlinear state estimation of a degrading battery system. Natick, Massachusetts: The MathWorks Inc.

Moura, S. J., Stein, J. L., \& Fathy, H. K. (2013). Battery-health conscious power management in plugin hybrid electric vehicles via electrochemical modeling and stochastic control. IEEE Transactions on Control Systems Technology, 21(3), 679-694. doi: 10.1109/TCST.2012.2189773

Onori, S., Serrao, L., \& Rizzoni, G. (2016). Hybrid electric vehicles. Springer London. doi: 10.1007/978-1-44716781-5

Özbek, M., Wang, S., Marx, M., \& Söffker, D. (2013). Modeling and control of a pem fuel cell system: A prac- 
tical study based on experimental defined component behavior. Journal of Process Control, Vol. 23, No. 3, 282-293.

Remmlinger, J., Buchholz, M., Meiler, M., Bernreuter, P., \& Dietmayer, K. (2011). State-of-health monitoring of lithium-ion batteries in electric vehicles by on-board internal resistance estimation. Journal of Power Sources, 196(12), 5357-5363. doi: 10.1016/j.jpowsour.2010.08.035

Ruiz, V., Pfrang, A., Kriston, A., Omar, N., den Bossche, P. V., \& Boon-Brett, L. (2018). A review of international abuse testing standards and regulations for lithium ion batteries in electric and hybrid electric vehicles. Renewable and Sustainable Energy Reviews, 81, 1427-1452. doi: 10.1016/j.rser.2017.05.195

Silvas, E., Hofman, T., Murgovski, N., Etman, L. F. P., \& Steinbuch, M. (2017). Review of optimization strategies for system-level design in hybrid electric vehicles. IEEE Transactions on Vehicular Technology, 66(1), 5770. doi: 10.1109/TVT.2016.2547897

Wang, Y., Sun, Z., \& Chen, Z. (2019). Rule-based energy management strategy of a lithium-ion battery, supercapacitor and PEM fuel cell system. Energy Procedia, 158, 2555-2560. doi: 10.1016/j.egypro.2019.02.003

Yue, M., Jemei, S., Gouriveau, R., \& Zerhouni, N. (2019). Review on health-conscious energy management strategies for fuel cell hybrid electric vehicles: Degradation models and strategies. International Journal of Hydrogen Energy, 44(13), 6844-6861. doi: 10.1016/j.ijhydene.2019.01.190

Zhang, P., Yan, F., \& Du, C. (2015). A comprehensive analysis of energy management strategies for hybrid electric vehicles based on bibliometrics. Renewable and Sustainable Energy Reviews, 48, 88-104. doi: 10.1016/j.rser.2015.03.093

\section{BIOGRAPHIES}

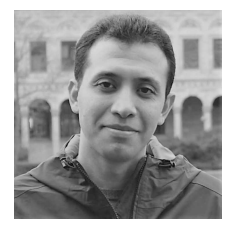

Ahmed M. Ali received the B.Sc. degree and the M.Sc. degree, both in Mechanical Engineering from the Military Technical College, Cairo, Egypt, in 2008 and 2015 respectively. He is now with the Chair of Dynamics and Control, University of Duisburg-Essen, where he works towards the Ph.D. degree in the field of optimal design and control of hybrid vehicles.

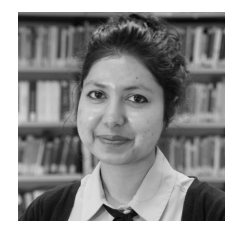

Bedatri Moulik received her Bachelors degree in Electrical Engineering and Masters degree in Control and Instrumentation Engineering from West Bengal University of Technology, Kolkata, India. She received her Dr.-Ing. degree from the Chair of Dynamics and Control, University of Duisburg-Essen, Germany. She is now an Assistant Professor at Amity University, Noida, India in the Department of Electrical and Electronics Engineering.

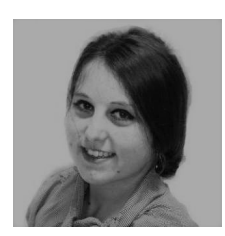

Nejra Beganovic received the B.Sc. and M.Sc. in Electrical Engineering from the University of Sarajevo, Chair of $\mathrm{Au}-$ tomatic Control and Electronics, BosniaHerzegovina, in 2009 and 2011 respectively. In 2016, she has received the Dr.-Ing. degree from the University of Duisburg-Essen, Chair of Dynamics and Control, Germany. From October 2017 to February 2019, she was an Assistant Professor at the International Burch University, Sarajevo. Since February 2019, she is a postdoctoral researcher at Mid-Sweden University, Department of Electronics Design, Sweden. Her research interests include structural health monitoring, lifetime prognostics, and advanced signal processing methods with special emphasis on real-time implementation of classification and prognostic algorithms.

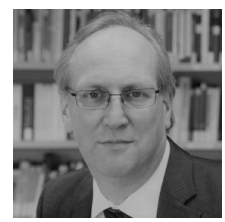

Dirk Söffker received the Dr.-Ing. degree in mechanical engineering and the Habilitation degree in automatic control/safety engineering from University of Wuppertal, Wuppertal, Germany, in 1995 and 2001, respectively. Since 2001, he leads the Chair of Dynamics and Control with the Engineering Faculty, University of Duisburg-Essen, Germany. His current research interests include elastic mechanical structures, modern methods of control theory, human interaction with safety systems, safety and reliability control engineering of technical systems, and cognitive technical systems. 